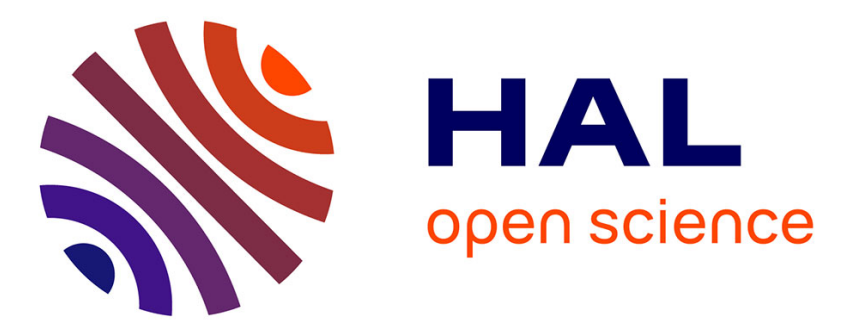

\title{
Relativistic electron scattering by magnetosonic waves: Effects of discrete wave emission and high wave amplitudes
}

A. V. Artemyev, Didier Mourenas, O. V. Agapitov, Vladimir Krasnoselskikh

\section{- To cite this version:}

A. V. Artemyev, Didier Mourenas, O. V. Agapitov, Vladimir Krasnoselskikh. Relativistic electron scattering by magnetosonic waves: Effects of discrete wave emission and high wave amplitudes. Physics of Plasmas, 2015, 22, pp.062901. 10.1063/1.4922061 . insu-01255790

HAL Id: insu-01255790

https://hal-insu.archives-ouvertes.fr/insu-01255790

Submitted on 14 Jan 2016

HAL is a multi-disciplinary open access archive for the deposit and dissemination of scientific research documents, whether they are published or not. The documents may come from teaching and research institutions in France or abroad, or from public or private research centers.
L'archive ouverte pluridisciplinaire HAL, est destinée au dépôt et à la diffusion de documents scientifiques de niveau recherche, publiés ou non, émanant des établissements d'enseignement et de recherche français ou étrangers, des laboratoires publics ou privés.

\section{(ㅇ)(1) $\$$}

Distributed under a Creative Commons Attribution - NonCommercial - NoDerivatives $\mid 4.0$ 


\section{AIP $\left.\right|_{\text {Physics of }}$ \\ Plasmas}

\section{Relativistic electron scattering by magnetosonic waves: Effects of discrete wave emission and high wave amplitudes}

A. V. Artemyev, D. Mourenas, O. V. Agapitov, and V. V. Krasnoselskikh

Citation: Physics of Plasmas 22, 062901 (2015); doi: 10.1063/1.4922061

View online: http://dx.doi.org/10.1063/1.4922061

View Table of Contents: http://scitation.aip.org/content/aip/journal/pop/22/6?ver=pdfcov

Published by the AIP Publishing

\section{Articles you may be interested in}

Probability of relativistic electron trapping by parallel and oblique whistler-mode waves in Earth's radiation belts Phys. Plasmas 22, 112903 (2015); 10.1063/1.4935842

Stability of relativistic electron trapping by strong whistler or electromagnetic ion cyclotron waves

Phys. Plasmas 22, 082901 (2015); 10.1063/1.4927774

Resonance zones for electron interaction with plasma waves in the Earth's dipole magnetosphere. II. Evaluation for oblique chorus, hiss, electromagnetic ion cyclotron waves, and magnetosonic waves

Phys. Plasmas 17, 042903 (2010); 10.1063/1.3310835

Electron inertia effect on small amplitude solitons in a weakly relativistic two-fluid plasma

Phys. Plasmas 12, 052103 (2005); 10.1063/1.1894398

Pitch-angle diffusion of relativistic electrons due to resonant interactions with whistler waves

Phys. Plasmas 6, 4597 (1999); 10.1063/1.873747

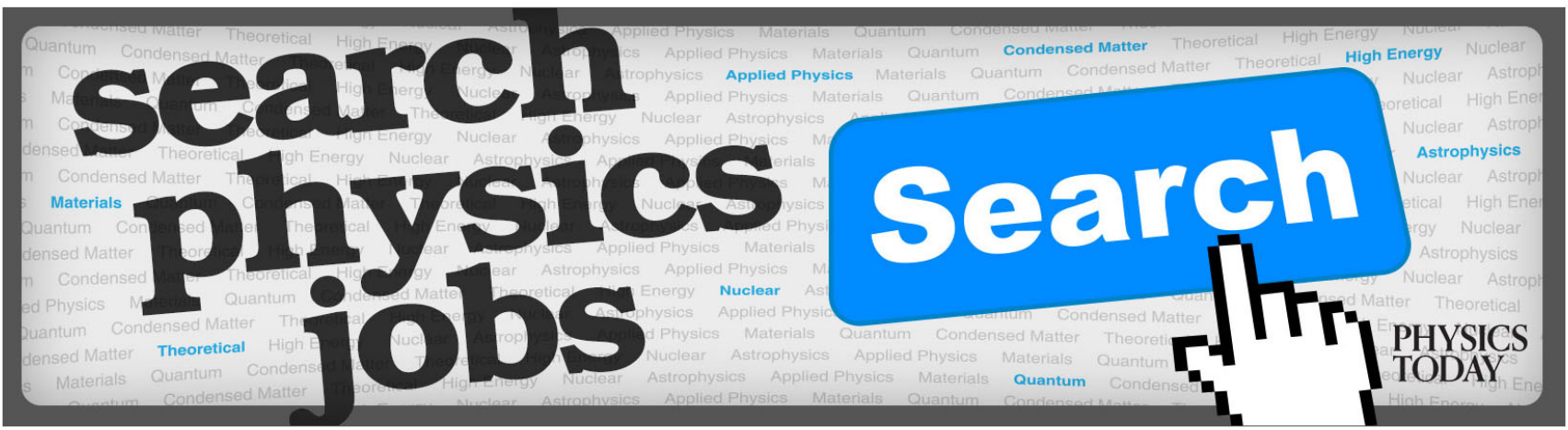




\title{
Relativistic electron scattering by magnetosonic waves: Effects of discrete wave emission and high wave amplitudes
}

\author{
A. V. Artemyev, ${ }^{1, a)}$ D. Mourenas, ${ }^{2}$ O. V. Agapitov, ${ }^{3, b)}$ and V. V. Krasnoselskikh ${ }^{2}$ \\ ${ }^{1}$ Space Research Institute, RAS, Moscow, Russia \\ ${ }^{2}$ LPC2E/CNRS - University of Orleans, Orleans, France \\ ${ }^{3}$ Space Sciences Laboratory, University of California, Berkeley, California 94720, USA
}

(Received 18 March 2015; accepted 18 May 2015; published online 3 June 2015)

\begin{abstract}
In this paper, we study relativistic electron scattering by fast magnetosonic waves. We compare results of test particle simulations and the quasi-linear theory for different spectra of waves to investigate how a fine structure of the wave emission can influence electron resonant scattering. We show that for a realistically wide distribution of wave normal angles $\theta$ (i.e., when the dispersion $\delta \theta \geq 0.5^{\circ}$ ), relativistic electron scattering is similar for a wide wave spectrum and for a spectrum consisting in well-separated ion cyclotron harmonics. Comparisons of test particle simulations with quasi-linear theory show that for $\delta \theta>0.5^{\circ}$, the quasi-linear approximation describes resonant scattering correctly for a large enough plasma frequency. For a very narrow $\theta$ distribution (when $\delta \theta \sim 0.05^{\circ}$ ), however, the effect of a fine structure in the wave spectrum becomes important. In this case, quasi-linear theory clearly fails in describing accurately electron scattering by fast magnetosonic waves. We also study the effect of high wave amplitudes on relativistic electron scattering. For typical conditions in the earth's radiation belts, the quasi-linear approximation cannot accurately describe electron scattering for waves with averaged amplitudes $>300 \mathrm{pT}$. We discuss various applications of the obtained results for modeling electron dynamics in the radiation belts and in the Earth's magnetotail. (C) 2015 AIP Publishing LLC.

[http://dx.doi.org/10.1063/1.4922061]
\end{abstract}

\section{INTRODUCTION}

Magnetosonic waves are widespread in the near-earth plasma environment where these waves are responsible for the scattering and acceleration of relativistic electrons in the radiation belts ${ }^{18}$ and magnetotail region, ${ }^{8,56}$ and at the bow shock. $^{23,55}$ Modern spacecraft observations suggest that amplitudes of fast magnetosonic waves ${ }^{35,50}$ and the corresponding wave occurrence rate ${ }^{28,29,31,34,43}$ are large enough for these waves to have a significant impact on the overall dynamics of relativistic electrons. Being excited by unstable ion distributions, ${ }^{29,57}$ fast magnetosonic waves can act as intermediaries in an energy transfer between high-energy ions and relativistic electrons (in contrast to electron whistler waves believed to be generated by an anisotropic electron population $^{14,53}$ ).

Spacecraft observations and numerical simulations demonstrate that fast magnetosonic emissions remain generally trapped within the near-equatorial region in the radiation belts ${ }^{17,21,38}$ where these waves could be an important agent for scattering electrons into Earth's atmosphere. ${ }^{30}$ They could also be partly responsible for important energetic electron injections recently observed deep inside the plasmasphere during substorms. ${ }^{51}$

The general approach for modeling relativistic electron scattering by fast magnetosonic waves consists in the application of the quasi-linear approximation, ${ }^{31,43,52}$ although several limitations of this approach were indicated in Refs. 7

\footnotetext{
${ }^{a)}$ Electronic mail: ante0226@gmail.com

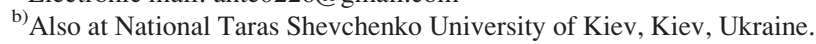

and 24. This approach assumes that wave emission is broad enough in the frequency $\omega$ space. However, theoretical models of the generation of magnetosonic waves show that these waves are exited at ion cyclotron harmonics. ${ }^{15,57}$ Thus, in reality, we deal with a finely structured wave emission instead of a broad structureless spectrum. Consequently, the applicability of the quasi-linear theory needs to be reexamined for such finely structured emissions. To perform the corresponding analysis, we use in this paper the test particle approach and compare the obtained results with a model of quasi-linear diffusion rates. $^{31}$

Fast magnetosonic waves propagate at a large angle relative to the background magnetic field, ${ }^{21}$ i.e., the corresponding wave vector $\mathbf{k}$ has a preponderant transverse component. Due to the low frequency (relative to the electron gyrofrequency $\Omega_{c e}$ ) of magnetosonic waves, only Landau resonance (when the parallel electron velocity becomes equal to the parallel wave phase velocity) is available for electrons with energies less than $5 \mathrm{MeV}$. Thus, the principal role during electron resonant interaction with magnetosonic waves is played by the parallel (along field lines) distribution of wave parameter. However, the strong localization of the wave emission along field lines allows to consider resonant interaction rather independently of the geomagnetic field configuration. This allows to apply the obtained results both for radiation belts with a dipolar magnetic field and for the magnetotail's magnetic field configuration with stretched field lines (where strong magnetosonic waves are often observed, see Ref. 56). Moreover, in the Earth's magnetotail, the ratio of plasma frequency $\Omega_{p e}$ to the local electron gyrofrequency 
is larger than the corresponding ratio in the radiation belts. It has been shown previously that the quasi-linear theory can be used for the description of electron interaction with magnetosonic waves only for short enough wavelength (i.e., for $\Omega_{p e} / \Omega_{c e}>5$, see Ref. 24). Thus, in this paper, we consider the general question of electron resonant interaction with finely structured fast magnetosonic waves for eventual application to both the radiation belts and the magnetotail physics.

\section{WAVE MODEL}

We consider whistler-mode waves with so low frequency $\omega$ that the ion contribution to wave dispersion becomes significant (or even dominant). The cold plasma dispersion relation of such waves is ${ }^{16,19}$

$$
\omega=\frac{\sqrt{\nu} \Omega_{c e}}{\sqrt{1+\left(\Omega_{p e} / k c\right)^{2}}},
$$

where $\nu=m_{e} / m_{i}$ and $m_{i}$ is the effective mass of the ion mixture. The cold plasma dispersion relation is valid when the cold electron and ion species are largely dominant over dilute hot species, as it is usually the case, for example, in the inner magnetosphere [e.g., see Ref. 26]. Moreover, the cold ion temperature is assumed to be so small that the corresponding thermal velocity is much smaller than $\sqrt{\nu}$ times the Alfven velocity (e.g., in the radiation belts the Alfven velocity is $\sim 100$ times larger than the thermal velocity of the cold ion component). In this case, cold ion thermal effects can be neglected even for waves propagating near the local ion cyclotron harmonics.

The corresponding refractive index $N$ is

$$
N^{2}=\frac{\Omega_{p e}^{2}}{\nu \Omega_{c e}^{2}}\left(1-\frac{\omega^{2}}{\nu \Omega_{c e}^{2}}\right)^{-1}=\frac{1}{\nu} \frac{\omega_{p e}^{2}}{1-\nu \omega_{i}^{2}},
$$

where we introduce new parameters $\omega_{i}=\omega /\left(\nu \Omega_{c e}\right)$ and $\omega_{p e}=\Omega_{p e} / \Omega_{c e}$. For a dipolar magnetic field, $\omega_{p e}$ can be written as a simple function of $L$-shell in the inner magnetosphere $^{40}$ (i.e., as a function of the distance $L R_{E}$ from the Earth measured in Earth radii $R_{E}$ ).

We use expressions for the electromagnetic field of the waves in the following form: ${ }^{45}$

$$
\begin{aligned}
& \mathbf{E}=E_{w, x} \sin \phi-E_{w, y} \cos \phi+E_{w, z} \sin \phi, \\
& \mathbf{B}=B_{w, x} \cos \phi-B_{w, y} \sin \phi+B_{w, z} \cos \phi,
\end{aligned}
$$

where for fast magnetosonic waves with $\theta \sim \pi / 2$, the relationships between electric and magnetic wave amplitude components are

$$
\begin{aligned}
& \frac{E_{w, x}}{B_{w, y}}=\frac{P-N^{2}}{P N \cos \theta}, \\
& \frac{E_{w, y}}{B_{w, y}}=\frac{D}{S-N^{2}} \frac{P-N^{2}}{P N \cos \theta},
\end{aligned}
$$

$$
\begin{aligned}
& \frac{E_{w, z}}{B_{w, y}}=-\frac{N}{P}, \\
& \frac{B_{w, x}}{B_{w, y}}=-\frac{D}{S-N^{2}} \frac{P-N^{2}}{P}, \\
& \frac{B_{w, z}}{B_{w, y}}=\frac{D}{S-N^{2}} \frac{P-N^{2}}{P} \tan \theta,
\end{aligned}
$$

and $P, S, D$, are Stix's coefficients. ${ }^{44}$ The wave phase is $\phi=k_{x} x+k_{z} z-\omega t \approx k(x+z \cos \theta)-\omega t$. Electromagnetic fields (3) can be expressed through two components of the vector potential $\mathbf{A}=-a_{y} \sin \phi \mathbf{e}_{x}+a_{x} \cos \phi \mathbf{e}_{x}$ and scalar potential $\Phi=\phi \cos \phi$. The relationship between amplitudes of wave components gives the relation between $a_{x}, a_{y}$, and $\phi$

$$
\begin{aligned}
\phi & =-\frac{1}{N} \frac{N^{2}}{P-N^{2}} \frac{S-N^{2}}{D} a_{y}, \\
a_{x} & =\frac{P}{P-N^{2}} \frac{S-N^{2}}{D} a_{y} .
\end{aligned}
$$

We introduce $B_{w}$ as the full wave magnetic field amplitude, i.e.,

$$
\frac{1}{2 \pi} \int_{0}^{2 \pi}|\mathbf{B}|^{2} d \phi=B_{w}^{2}
$$

Thus, for $\theta \sim \pi / 2$, we have the corresponding expression for $B_{w, y}$

$$
B_{w, y}=B_{w}\left(\frac{D}{S-N^{2}} \frac{P-N^{2}}{P}\right)^{-1} \cos \theta .
$$

The combination of Eqs. (5) and (6) gives

$$
\begin{aligned}
A_{y} & =\left(B_{w} / k\right) \sin \phi, \\
A_{x} & =\left(B_{w} / k\right) a \cos \phi, \\
\Phi & =\left(B_{w} / N k\right) a \cos \phi, \\
a & =\frac{P}{P-N^{2}} \frac{S-N^{2}}{D} .
\end{aligned}
$$

Fig. 1 shows the coefficient $a$ as a function of wave frequency $\omega_{i}$ for the simplified dispersion relation (1) and when

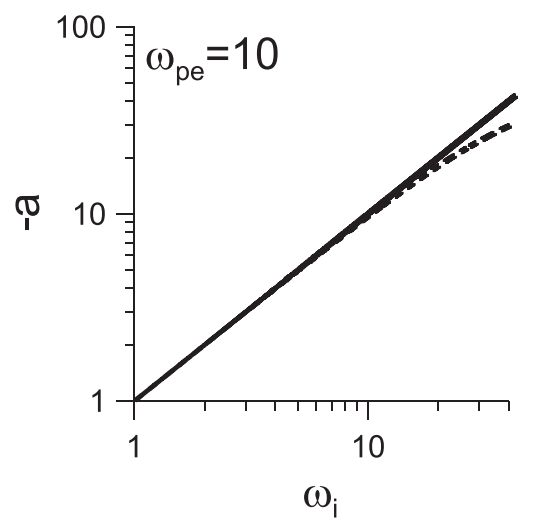

FIG. 1. Dependence of parameter $a$ on the normalized wave frequency $\omega_{i}=\omega /\left(\nu \Omega_{c e}\right)$. The solid line shows results for the simplified dispersion relation (1), while the dotted line shows results obtained with the full dispersion relation of whistler-mode waves. ${ }^{16}$ 
using the full dispersion relation of whistler-mode waves. ${ }^{16}$ One can see that for $\omega_{i}<20$, we can safely use only the simplified dispersion. One additional property of coefficient $a$ can be derived by taking into account that $N^{2} \gg S$ and $N^{2} \ll P$ for magnetosonic waves. Accordingly, Eq. (7) gives $a \approx-N^{2} / D \approx-N^{2} \Omega_{c e} \omega / \Omega_{p e}^{2}$. This ratio does not depend on plasma frequency (see Eq. (2)). Thus, the parameter $a$ remains more or less the same as plasma frequency (i.e., $\left.\omega_{p e}\right)$ varies.

\section{HAMILTONIAN EQUATIONS}

The motion of relativistic electrons with charge $e$ and mass $m$ in the background magnetic field $B_{0}(z)$ and wave field (3) can be described by the following Hamiltonian:

$$
\begin{aligned}
H & =\sqrt{m^{2} c^{4}+p_{z}^{2} c^{2}+\left(c p_{x}+e A_{x}\right)^{2}+\left(e A_{y, \Sigma}\right)^{2}}-e \Phi \\
e A_{y, \Sigma} & =e A_{y}+e x B_{0}(z)
\end{aligned}
$$

where $A_{x}, A_{y}$, and $\Phi$ are given by Eq. (7). We use magnetic field model $B_{0}(z)=B_{e q} b\left(z / R_{0}\right)$, where $b\left(z / R_{0}\right)$ is a dipole magnetic field with $R_{0}=L R_{E}$ ( $L$-shell and Earth radius $R_{E}$ ). The corresponding equatorial gyrofrequency is $\Omega_{0}=e B_{e q} / m c$. We introduce dimensionless variables and parameters

$$
\begin{gathered}
H_{0} / m c^{2} \rightarrow H_{0}, \quad \mathbf{p} / m c \rightarrow \mathbf{p}, \quad \mathbf{r} / R_{0} \rightarrow R_{0}, \\
t c / R_{0} \rightarrow t, \quad \chi=\Omega_{0} R_{0} / c, \quad b_{w}=e B_{w} / k m c^{2}, \\
k \Omega_{0} / c \rightarrow k, \quad \omega / \Omega_{0} \rightarrow \omega, \quad N \rightarrow(k / \omega),
\end{gathered}
$$

and rewrite the Hamiltonian (8) as

$$
\begin{aligned}
H= & -b_{w}(a / N) \cos \phi \\
& +\sqrt{1+p_{z}^{2}+\left(p_{x}+b_{w} a \cos \phi\right)^{2}+\left(b_{w} \sin \phi+\chi x b\right)^{2}} .
\end{aligned}
$$

The parameter $b_{w}$ is small. Thus, the Hamiltonian (10) can be expanded as

$$
\begin{aligned}
H & =H_{0}+b_{w}\left(\frac{p_{x}}{H_{0}} a \cos \phi+\frac{\chi x b}{H_{0}} \sin \phi-\frac{a}{N} \cos \phi\right), \\
H_{0} & =\sqrt{1+p_{z}^{2}+p_{x}^{2}+(\chi x b)^{2}} .
\end{aligned}
$$

The Hamiltonian $H_{0}$ shows that the unperturbed motion (for $\left.b_{w}=0\right)$ inside the phase plane $\left(x, p_{x}\right)$ is periodic. Thus, we can introduce the adiabatic invariant (i.e., magnetic moment)

$$
I_{x}=\frac{1}{2 \pi} \oint p_{x} d x=\frac{H_{0}^{2}-1-p_{z}^{2}}{2 \chi b} .
$$

The corresponding change of variables is

$$
\begin{aligned}
x & =\sqrt{2 I_{x} / \chi b} \sin \beta, \\
p_{x} & =\sqrt{2 I_{x} \chi b} \cos \beta, \\
\dot{\beta} & =-\chi b / H_{0},
\end{aligned}
$$

and the final Hamiltonian $H_{0}$ can be written as

$$
H_{0}=\gamma=\sqrt{1+p_{z}^{2}+2 \chi I_{x} b}
$$

We substitute Eqs. (13) and (14) into Eq. (11) and obtain

$$
\begin{aligned}
H= & H_{0}-b_{w} \frac{a}{N} \cos \phi \\
& +b_{w} \frac{\sqrt{2 I_{x} \chi b}}{\gamma}(a \cos \phi \cos \beta+\sin \beta \sin \phi) .
\end{aligned}
$$

The corresponding wave phase is

$$
\phi=\chi\left(k_{z} z+k_{x} \sqrt{2 I_{x} / \chi b} \sin \beta-\omega t\right) .
$$

We use classical expansions of $\cos \phi, \sin \phi$ in Bessel functions $J_{n}$ to get the series

$$
\begin{aligned}
\sin \phi \sin \beta & =-\sum_{n=-\infty}^{\infty} J_{n}^{\prime}(\eta) \cos (\tilde{\phi}+n \beta), \\
\cos \phi \cos \beta & =\sum_{n=-\infty}^{\infty} \frac{n}{\eta} J_{n}(\eta) \cos (\tilde{\phi}+n \beta), \\
\cos \phi & =\sum_{n=-\infty}^{\infty} J_{n}(\eta) \cos (\tilde{\phi}+n \beta),
\end{aligned}
$$

where

$$
\begin{aligned}
\tilde{\phi} & \approx \chi(k z \cos \theta-\omega t), \\
\eta & \approx k \sqrt{2 I_{x} \chi / b} .
\end{aligned}
$$

Substituting Eq. (17) into Eq. (15), we get

$$
\begin{gathered}
H=H_{0}-b_{w} \sum_{n=-\infty}^{\infty} W_{n} \cos (\tilde{\phi}+n \beta), \\
W_{n}=\frac{\sqrt{2 I_{x} \chi b}}{\gamma}\left(J_{n}^{\prime}(\eta)-\frac{a n}{\eta} J_{n}(\eta)\right)+\frac{a}{N} J_{n}(\eta) .
\end{gathered}
$$

In the following, we consider only the Landau resonance $n=0$, which is known to be the most effective resonance for electron interaction with magnetosonic waves (see Refs. 18, 31, and 43). Thus, the Hamiltonian (19) takes the form

$$
\begin{gathered}
H=H_{0}-b_{w} W_{0} \cos (\tilde{\phi}), \\
W_{0}=\frac{a}{N} J_{0}(\eta)-\frac{\sqrt{2 I_{x} \chi b}}{\gamma} J_{1}(\eta),
\end{gathered}
$$

where $J_{0}^{\prime}=-J_{1}$. Hamiltonian equations for the Hamiltonian (20) are

$$
\left\{\begin{array}{l}
\dot{z}=p_{z} / \gamma \\
\dot{p}_{z} \approx-\left(w_{0} b^{\prime}(z) / 2 \gamma\right)+F \\
F=\chi b_{w} k W_{0} \cos \theta \sin \tilde{\phi} \\
\tilde{\phi}=\chi(k z \cos \theta-\omega t), \quad \eta=k \sqrt{w_{0} / b} \\
k W_{0}=a \omega J_{0}(\eta)-k \gamma^{-1} \sqrt{w_{0} b} J_{1}(\eta),
\end{array}\right.
$$

where we introduced $w_{0}=\left(\gamma_{0}^{2}-1\right) \sin ^{2} \alpha_{0}=2 \chi I_{x}, \gamma_{0}$ is the initial Lorentz factor and $\alpha_{0}$ the initial equatorial pitch-angle. 
In Eq. (21), $k$ is determined by Eq. (2). When many waves are present in the system, the force $F$ takes the following form:

$$
\begin{aligned}
F & =\sum_{l} \chi b_{w, l} k_{l} W_{0, l} \cos \theta_{l} \sin \tilde{\phi}_{l}, \\
\tilde{\phi} & =\chi\left(k_{l} z \cos \theta-\omega_{l} t\right), \quad \eta_{l}=k_{l} \sqrt{w_{0} / b}, \\
k_{l} W_{0, l} & =a_{l} \omega_{l} J_{0}\left(\eta_{l}\right)-k_{l} \gamma^{-1} \sqrt{w_{0} b} J_{1}\left(\eta_{l}\right) .
\end{aligned}
$$

\section{TEST PARTICLE MODEL VS. QUASI-LINEAR APPROXIMATION}

To solve system (22) numerically, we first need to define the wave distribution over angles of propagation $\theta$ and frequencies $\omega$. We use here the approach proposed in Ref. 47: the wave intensity spectrum is given by equation

$$
P \sim \exp \left(-\frac{\left(\omega-\omega_{m}\right)^{2}}{\delta \omega^{2}}-\frac{\left(\theta-\theta_{m}\right)^{2}}{\delta \theta^{2}}\right)
$$

while the corresponding wave amplitudes are

$$
\begin{aligned}
B_{w, l} & =\frac{B_{w 0}}{\Sigma_{B}} \exp \left(-\frac{\left(\omega_{l}-\omega_{m}\right)^{2}}{2 \delta \omega^{2}}-\frac{\left(\theta_{l}-\theta_{m}\right)^{2}}{2 \delta \theta^{2}}\right), \\
\Sigma_{B} & =\frac{1}{\sqrt{2}} \sum_{l=1}^{N_{l}} \exp \left(-\frac{\left(\omega_{l}-\omega_{m}\right)^{2}}{2 \delta \omega^{2}}-\frac{\left(\theta_{l}-\theta_{m}\right)^{2}}{2 \delta \theta^{2}}\right),
\end{aligned}
$$

where $B_{w 0}$ is root-mean-square (RMS) wave amplitude. Wave characteristics are determined according to spacecraft observations: ${ }^{18,31} \omega_{m}=7 \nu \Omega_{c e}, \delta \omega=2 \nu \Omega_{c e}, \theta_{m}=89^{\circ}, \delta \theta$ $=0.6^{\circ}$. We use 100 different values of $\theta_{l} \in\left[88.6^{\circ}, 89.2^{\circ}\right]$ and 100 different value of frequency $\omega_{l} \in\left[\omega_{m}-2 \delta \omega\right.$, $\left.\omega_{m}+2 \delta \omega\right]$. Thus, the total number of waves is $N_{l}=10^{4}$.

The distribution of wave intensity along field lines is set in agreement with spacecraft observation ${ }^{31} P \sim \exp \left(-\left(\lambda / 3^{\circ}\right)^{2}\right)$ for magnetic latitude $|\lambda|<3^{\circ}$, and $P=0$ for $|\lambda|>3^{\circ}$.

Two examples of particle trajectories calculated by solving numerically the system (22) are shown in Fig. 2. One bounce period contains two time intervals with variations of particle energy $\gamma$ and equatorial pitch-angle $\alpha_{0}$ : small amplitude variations correspond to the nonresonant passage of particles through the group of waves (when particles propagate in opposite direction relative to the waves), while significant variations of particle parameters correspond to efficient resonant scattering by the waves.

We use Eq. (22) to trace particle ensemble and calculate diffusion coefficients as ${ }^{27}$

$$
D_{\alpha \alpha}=\left(\Delta \alpha_{0}\right)^{2} / \tau_{b}, \quad D_{E E}=(\Delta E / E)^{2} / \tau_{b},
$$

where $\Delta \alpha_{0}$ and $\Delta E$ denote changes of particle equatorial pitch-angle and energy, respectively, while $E=m c^{2}\left(\gamma_{0}-1\right)$ is the initial particle energy, and $\tau_{b} \approx 0.085 \sqrt{1-\gamma_{0}^{-2}}$ $L\left(1.3-0.56 \sin \alpha_{0}\right)$ is the electron bounce period measured in seconds. ${ }^{27}$ To calculate diffusion coefficients (25) for given pitch-angle $\alpha_{0}$ and energy $E$, we numerically integrate $10^{3}$ trajectories and determine the jumps $\Delta \alpha_{0}$ and $\Delta E$ after one passage through the equatorial plane. Each trajectory is integrated during a time interval equal to half of the bounce period (particles are run from one mirror point and integration stops when these particles reach the second mirror point on the other side of the equator). For given $\alpha_{0}$ and energy $E$, all particles have the same initial velocities, but for integration of each trajectory, we generate the unique initial phases of waves approximating the spectrum. Then, we calculate the average over ensemble values of $\Delta \alpha_{0}$ and $\Delta E$. Comparing the latter diffusion coefficients obtained from test-particle simulations with coefficients derived in the framework of quasi-linear theory should allow us to determine the limits of applicability of the approximation of stochastic particle scattering by a broad wave ensemble. ${ }^{24,46,47}$ To obtain quasi-linear diffusion coefficients, we use the analytical model proposed and tested in Ref. 31. This model uses the dispersion relation (2) and the same parameters $\theta_{m}$, $\omega_{m}, \delta \theta, \delta \omega$ as the test particle model.

\section{A. Wide wave spectrum}

In this subsection, we compare quasi-linear diffusion coefficients with diffusion coefficients (25) obtained from test particle simulations. In the test particle simulations, we use the wave power distribution (23) and approximate it by $10^{4}$ waves with initially random phases distributed uniformly within the $[0,2 \pi]$ range. Fig. 3 shows the resulting diffusion coefficients. One can see that for $\omega_{p e}=10$, the quasi-linear theory gives results in very good agreement with test particle
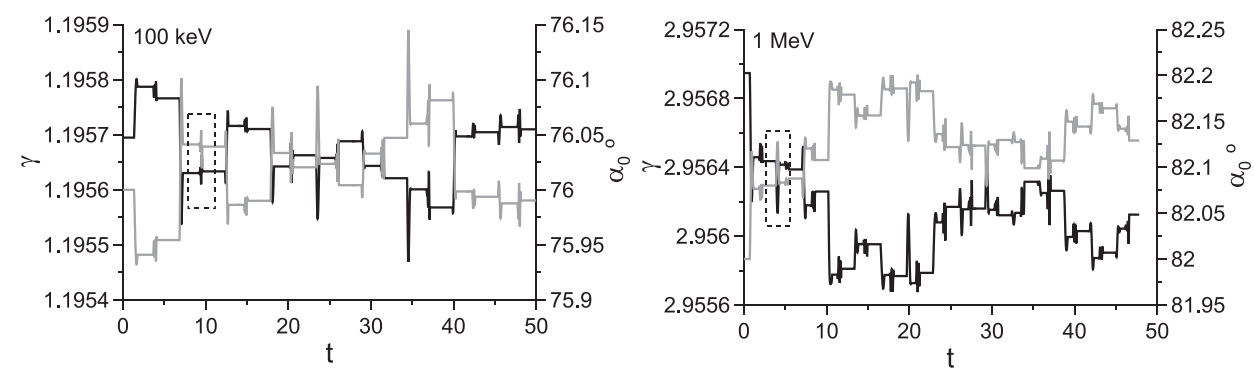

FIG. 2. Two examples of particle trajectories are displayed for $100 \mathrm{keV}$ and $1 \mathrm{MeV}$. Black curves show time profiles of $\gamma$, while grey curves show time profiles of equatorial pitch-angle $\alpha_{0}$. Dotted boxes show an interval of time corresponding to one bounce period. The background magnetic field corresponds to $L$-shell equal to $4.5, \omega_{p e}=4.5$, wave amplitude is $B_{w 0}=100 / \sqrt{2}$ pT. To integrate the trajectory for many bounce periods, we consider waves propagating in the same direction below and above the equatorial plane, i.e., $\cos \theta>0$. 

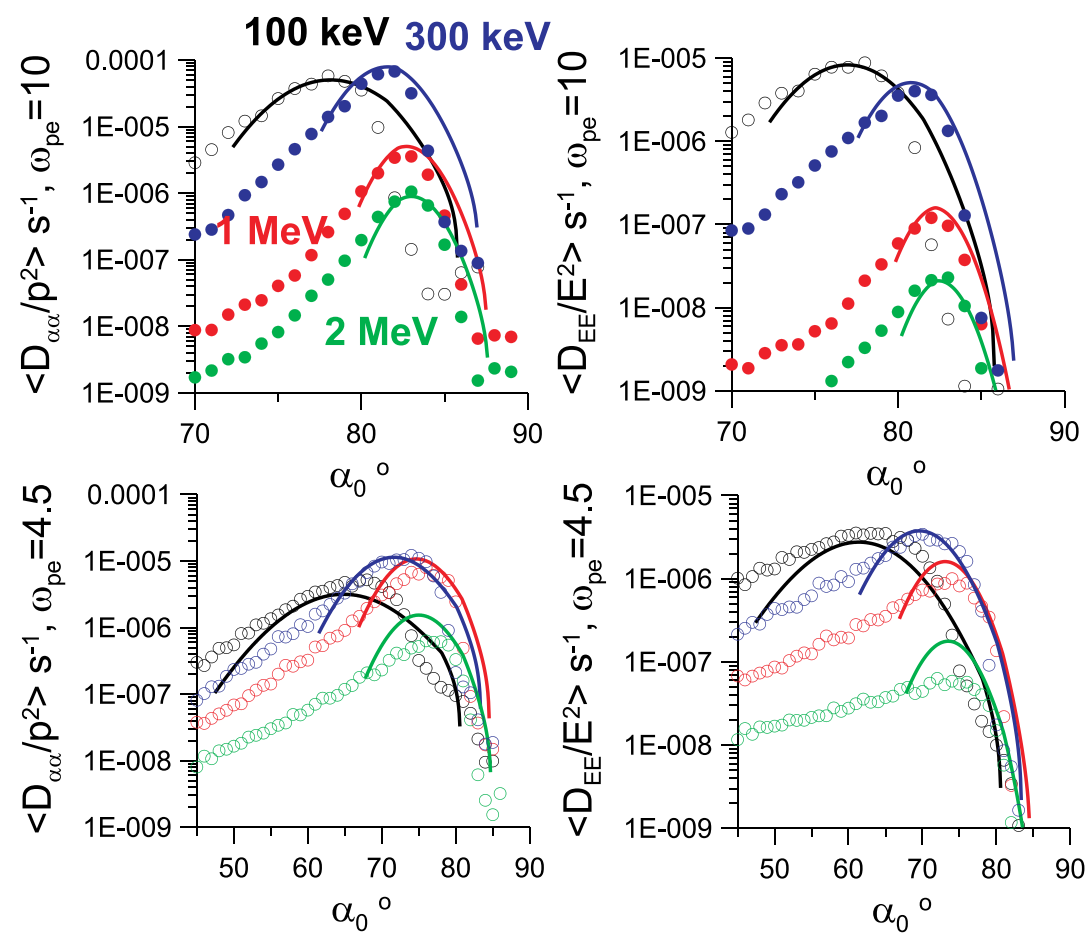

FIG. 3. Comparison of pitch-angle and diffusion rates obtained by test particle simulations (colored circles) and quasilinear approximation (colored curves). Three values of $\omega_{p e}$ parameter are used. The background magnetic field corresponds to $L$-shell equal to 4.5 , wave amplitude is $B_{w 0}=100 / \sqrt{2} \mathrm{pT}$.
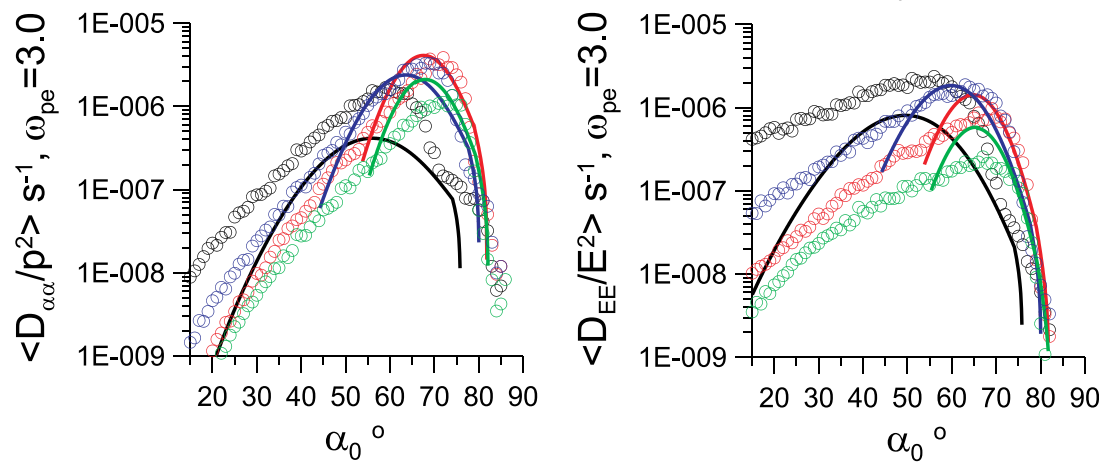

simulations (for both energy and pitch-angle scattering). However, as $\omega_{p e}$ becomes smaller, the discrepancy increases progressively.

This is an effect of the spatial confinement of magnetosonic waves inside a narrow latitudinal region close to the equatorial plane: if the wavelength becomes larger than the size of this domain (or, equivalently, if the time of resonant wave particle interaction becomes larger than the laps of time necessary for electrons to travel through this domain), particles can experience an additional transit time scattering even in the non-resonant case, violating a condition of applicability of the quasi-linear theory. ${ }^{7,24}$ This effect directly comes from the dispersion property of magnetosonic waves, i.e., from the dependence of the wavelength on plasma density.

As the results displayed in Fig. 3 demonstrate the validity of the quasi-linear approach to model electron scattering by magnetosonic waves for $\omega_{p e}=10$, we shall hereafter keep the same value of $\omega_{p e}$ to further investigate the possible effects of a discrete wave spectrum, which have not been studied before.

\section{B. Discrete wave spectrum: Resonance overlapping}

To investigate the effect of a discrete wave spectrum, we use the test particle approach with a modified distribution
(24): for the frequency distribution, we use only nine separate harmonics $\omega_{i} \in[3,11]$, while the distribution of $\theta$ angle remains the same as the one used to obtain results shown in Fig. 3. We perform calculations only for $\omega_{p e}=10$ and show the corresponding results in Fig. 4.

One can see that there is no significant difference between quasi-linear diffusion coefficients (calculated with the full spectrum (24)) and the results of test particle simulations obtained when considering a discrete spectrum of waves. This result stems from the relatively broad distribution of wave $\theta$ angles. For such a distribution, the resonances corresponding to separate ion cyclotron harmonics overlap in phase space ${ }^{10}$ and, thus, the resonant wave-particle interaction is well described in the framework of the quasi-linear approximation. ${ }^{39}$

The Landau resonance corresponds to the condition $\omega=\left(p_{z} / \gamma\right) k_{\|}$, where parallel wave phase velocity $\omega / k_{\|}$ $=\omega / k \cos \theta$ can be written as (see dispersion relation (1))

$$
\frac{\omega}{k_{\|}}=\frac{c \sqrt{\nu}}{\omega_{p e} \cos \theta}\left(1-\frac{\omega^{2}}{\nu \Omega_{c e}^{2}}\right)^{1 / 2} .
$$

Thus, the width of the resonance corresponding to a variance $\delta \theta$ can be calculated as (for small $\delta \theta$ ) 


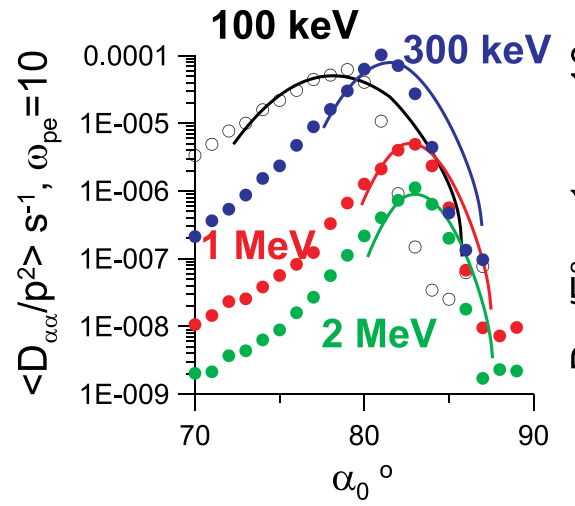

$$
\delta\left(\omega / k_{\|}\right)=\frac{\omega}{k_{\|}} \delta \theta \tan \theta_{m} .
$$

On the other hand, Eq. (26) gives a relation for the distance between two resonances as a function of ion cyclotron harmonic numbers $l$ and $l+1$ (i.e., with $\omega_{i}=l$ and $\omega_{i}=l+1$ )

$$
\Delta_{l}\left(\omega / k_{\|}\right)=\frac{\omega}{k_{\|}} \frac{\nu l}{1-\omega^{2} / \nu \Omega_{c e}^{2}} .
$$

Thus, the condition of overlapping of all $l$ resonances $\delta\left(\omega / k_{\|}\right)>l \Delta_{l}\left(\omega / k_{\|}\right)$can be written as

$$
\delta \theta>\frac{\nu l^{2} / \tan \theta_{m}}{1-\omega^{2} / \nu \Omega_{c e}^{2}} \approx \frac{2 \nu l^{2}}{\tan \theta_{m}} .
$$

For $l \epsilon^{3,11}$ and $\theta_{m}=89^{\circ}$, the condition (29) is satisfied for $\delta \theta>0.1^{\circ}$. Moreover, the condition of overlapping of two neighboring resonances $\delta\left(\omega / k_{\|}\right)>\Delta_{l}\left(\omega / k_{\|}\right)$is satisfied for $\delta \theta>0.01^{\circ}$. Thus, almost all realistic widths of the $\theta$ distribution should lead to stochastic scattering of charged particles by magnetosonic waves, even in the case of a discrete frequency spectrum.
FIG. 4. Comparison of pitch-angle diffusion rates obtained by test particle simulations (colored circles) and based on the quasi-linear approximation (colored curves). Wave spectrum consists in nine separate harmonics $\omega_{i} \in[3,11]$, while $\omega_{p e}=10$. The background magnetic field corresponds to a $L$-shell equal to 4.5 , wave amplitude is $B_{w 0}=100 / \sqrt{2} \mathrm{pT}$.
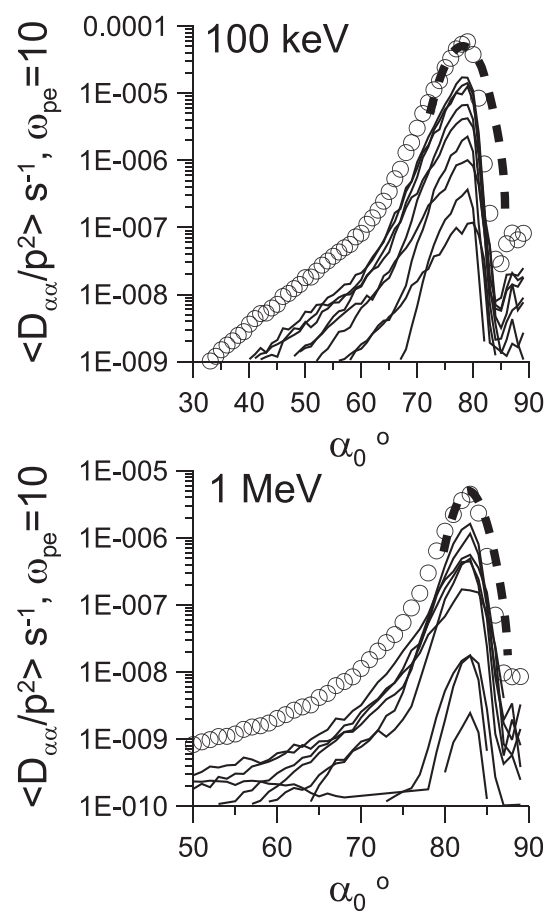
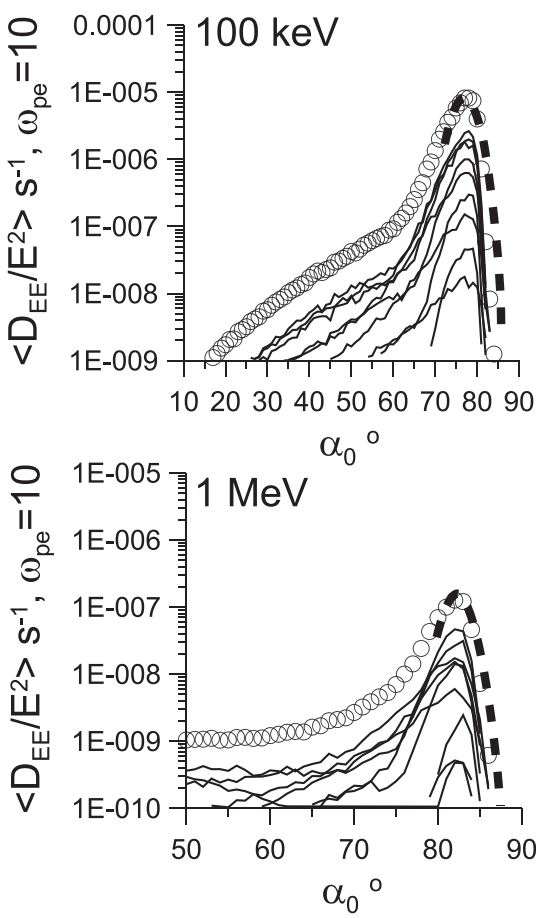

FIG. 5. Comparison of pitch-angle diffusion rates obtained by test particle simulations (circles and thin curves) and quasi-linear approximation (dotted curve). Thin curves show diffusion coefficients calculated for a given frequency $\omega_{i}=l$ with $l \in[3,11]$. Circles show the sum of individual diffusion coefficients. Two energies are used, while $\omega_{p e}=10$. The background magnetic field corresponds to a $L$-shell equal to 4.5 , wave amplitude is $B_{w 0}=$ $100 / \sqrt{2} \mathrm{pT}$. 

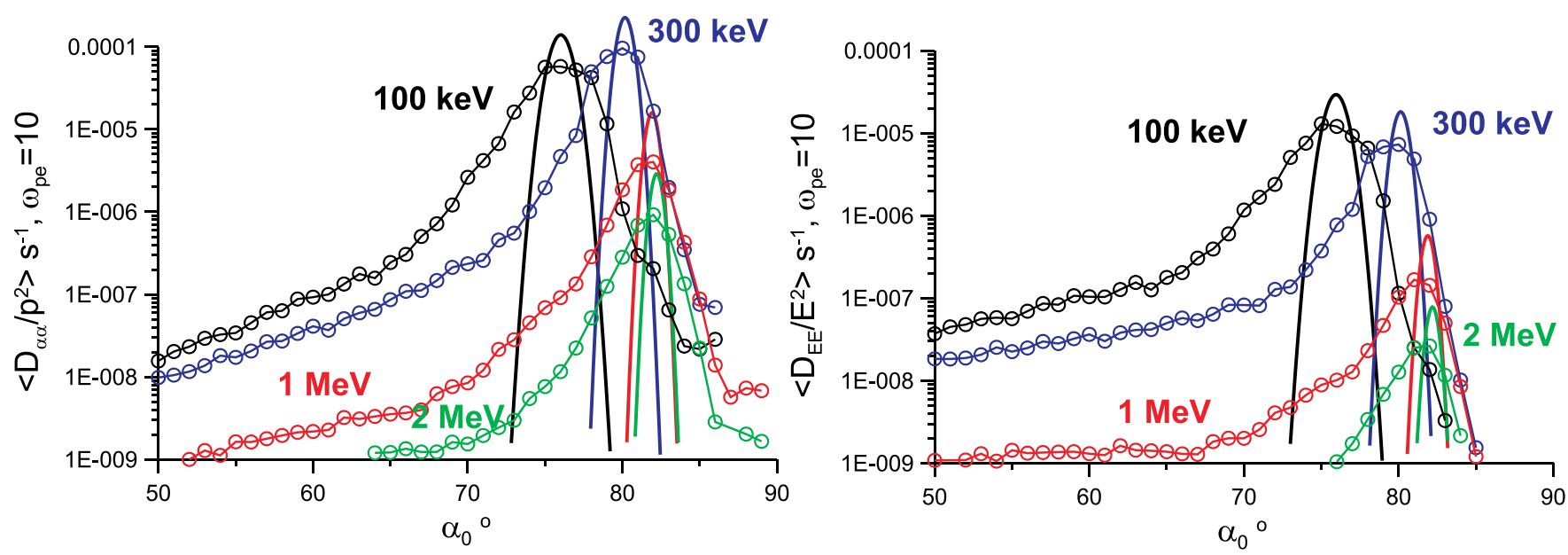

FIG. 6. Comparison of pitch-angle diffusion rates obtained by test particle simulations (thin curves with circles) and quasi-linear approximation (solid curve). Four energies are used, while $\omega_{p e}=10$ and a small $\delta \theta=0.06^{\circ}$. The background magnetic field corresponds to $L$-shell equal to 4.5 , wave amplitude is $B_{w 0}=$ $100 / \sqrt{2} \mathrm{pT}$.

compared with the quasi-linear approximation. Indeed, Fig. 6 shows a clear difference between the diffusion rates obtained for the broadband and discrete spectrum models. A discrete wave spectrum leads to diffusion rates with two-three times smaller maximum levels, but a much wider distribution over equatorial pitch-angles than within the quasi-linear approximation. Since the narrowness in equatorial pitch-angles of the diffusion peak is directly provided by the Landau resonance condition, it seems to imply that the significant diffusion outside of this domain obtained with test particle simulations in the case of a discrete spectrum should come from non-resonant (transit time) scattering. Thus, the applicability of quasi-linear theory for very small $\delta \theta$ remains questionable.

To further check the dependence of diffusion rate profiles on parameter $\delta \theta$, we plot in Fig. 7 the results of test particle simulations for several values of $\delta \theta$. Numerical modelling shows that the value of $\delta \theta$ determines both the position of the location of the maximum and the effective width (in pitch-angle domain) of diffusion rates.

It is worth noting the nonmonotoneous dependence of the diffusion rate maximum level on $\delta \theta$, e.g., for $100 \mathrm{keV}$, the minimum value of the pitch-angle diffusion rate can be

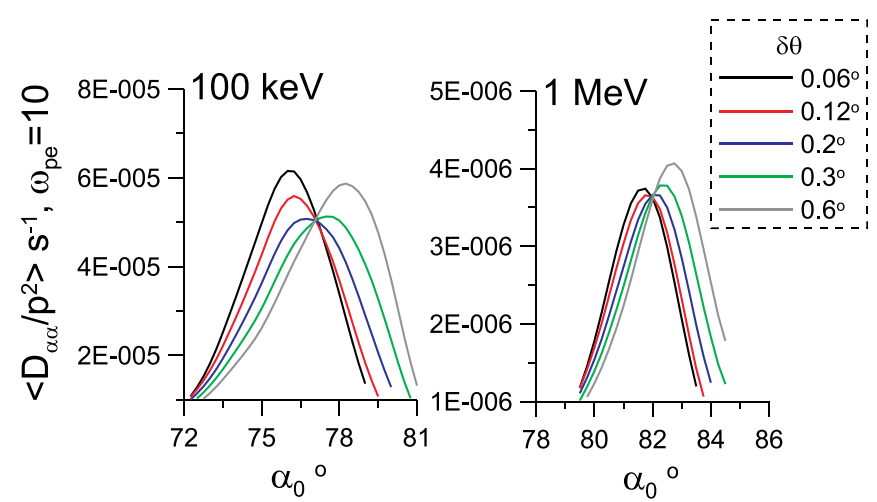

FIG. 7. Comparison of pitch-angle diffusion rates obtained by test particle simulations for different $\delta \theta$. Two energies are used, while $\omega_{p e}=10$. The background magnetic field corresponds to $L$-shell equal to 4.5 , wave amplitude is $B_{w 0}=100 / \sqrt{2} \mathrm{pT}$. found for $\delta \theta \sim 0.3^{\circ}$. Nevertheless, the variation with $\delta \theta$ of the (pitch-angle) position of the diffusion rate maximum can be explained in the frame of quasi-linear theory. In the very small $\delta \theta$ limit, the position of the maximum should be given by the Landau resonance condition at the mean angle $\theta=\theta_{m}$. However, when $\delta \theta$ becomes large enough, a slight shift to higher pitch-angles is allowed by the larger width of the $\theta$ distribution $P$ in Eq. (23), due to the rough proportionality of the quasi-linear diffusion rate to $\sim P(\theta) \tan ^{2} \alpha_{0}$ (see Ref. 31)-e.g., compare the locations of quasi-linear diffusion peaks in Figs. 4 and 6.

Although both Figs. 6 and 7 demonstrate some sensible differences between results of electron scattering obtained by test particle modeling and within the quasi-linear approximation, it should be stressed that these differences remain smaller than the accuracy of determination of actual wave parameters in real spacecraft observations. Thus, the loss of accuracy of the quasi-linear theory due to the effect of resonance splitting at very small $\delta \theta$ does not look as important as the effect of a small enough plasma density ${ }^{7,24}$ demonstrated before in Fig. 2.

The effect of a discrete wave spectrum can be further investigated in the case of more specific spectrum shapes. For example, we can assume that the spectrum contains two separate maxima at $\omega_{i}=\omega_{1}$ and $\omega_{i}=\omega_{2}$. Such a spectrum can be approximated as

$$
\begin{aligned}
P \sim & \exp \left(-\frac{\left(\theta-\theta_{m}\right)^{2}}{\delta \theta^{2}}\right) \\
& \times\left(\exp \left(-\frac{\left(\omega-\omega_{1}\right)^{2}}{\delta \omega^{2}}\right)+\exp \left(-\frac{\left(\omega-\omega_{2}\right)^{2}}{\delta \omega^{2}}\right)\right) .
\end{aligned}
$$

For a discrete spectrum approximation, we consider only two harmonics $\omega_{i}=\omega_{1,2}$ and 100 waves with different $\theta$ values. For the wide spectrum, we separate the distribution (31) into 100 frequencies $\omega_{i} \in[3,21]$ (in both cases, we use $\left.\omega_{1}=6, \omega_{2}=19\right)$. For a small enough $\delta \theta$, the separation between resonances occurring at $\omega_{1}$ and $\omega_{2}$ should a priori lead to some difference between $D_{\alpha \alpha}$ obtained for discrete or 
wide spectra. Fig. 8 shows indeed that we have two localized maxima in the case of a discrete spectrum. The deepness of the minimum in $D_{\alpha \alpha}$ located between these maxima decreases with increase of $\delta \theta$ : already for $\delta \theta=0.6^{\circ}$, the difference between $D_{\alpha \alpha}$ obtained with discrete and wide spectra disappears. This confirms again that the effect of the discreteness of the spectrum becomes important only for extremely small $\delta \theta$ values.

\section{Large wave amplitudes}

In this subsection, we check the applicability of the quasi-linear approximation for high-amplitude magnetosonic waves. To this aim, we use test particle simulations to obtain pitch-angle and energy diffusion coefficients for wave amplitudes $B_{w 0}$ ranging from $100 \mathrm{pT}$ up to $2.5 \mathrm{nT}$ (such very high amplitude waves were recently observed in the radiation belts and in the Earth magnetotail, see Refs. 50 and 56). Diffusion coefficients are normalized on $2\left(B_{w 0} / 100 \mathrm{pT}\right)^{2}$ to allow an easier comparison between the results obtained with different wave amplitudes. Therefore, if the normalized diffusion coefficients coincide with the coefficients obtained for $B_{w 0}=100 \mathrm{pT}$, then the quasi-linear approximation should work well for the considered wave amplitude. Fig. 9 shows that the quasi-linear approximation is applicable for $B_{w 0}<300 \mathrm{pT}$, while for larger wave amplitudes the results
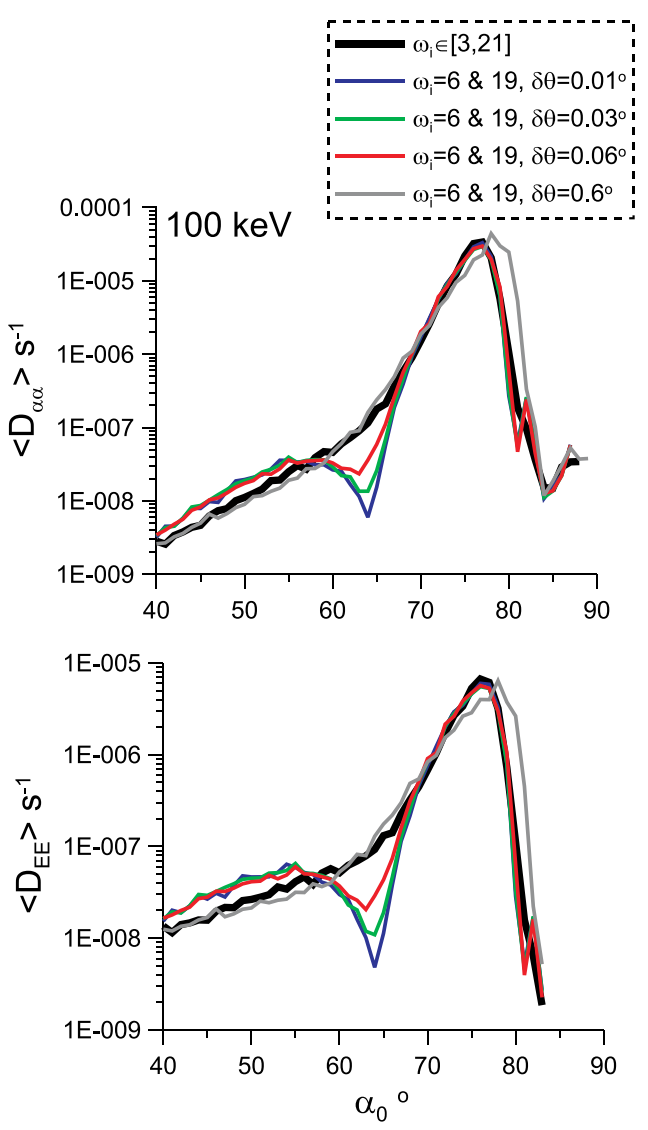

FIG. 8. Comparison of pitch-angle diffusion rates obtained by test particle simulations for discrete and wide spectra (electron energy is $100 \mathrm{keV}$, $\left.\omega_{p e}=10\right)$. The background magnetic field corresponds to $L$-shell equal to 4.5 , wave amplitude is $B_{w 0}=100 / \sqrt{2}$ pT. Results obtained for a wide spectrum are the same for all used values of $\delta \theta$.

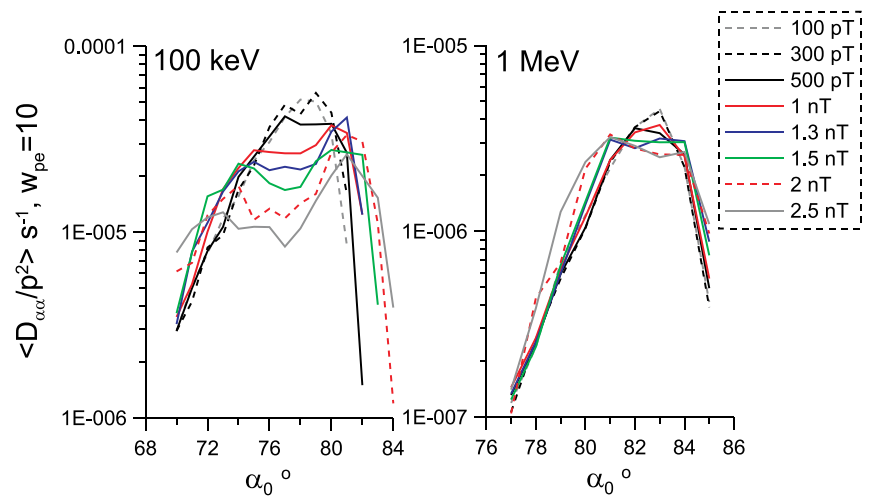

FIG. 9. Comparison of pitch-angle and diffusion rates obtained by test particle simulations for different wave amplitudes. Two energies are used, while $\omega_{p e}=10$. The background magnetic field corresponds to $L$-shell equal to 4.5. All diffusion rates are normalized on $\left(B_{w 0} / 100 \mathrm{pT}\right)^{2}$.

of test particle simulations do not coincide any more with the predictions of the quasi-linear theory. For large wave amplitudes, the diffusion coefficients increase weakly with $B_{w 0}$, while the quasi-linear theory predicts an increase proportional to $\sim B_{w 0}^{2}$. Similar results have been obtained when considering high amplitude parallel chorus waves (see Ref. 47).

The inapplicability of the quasi-linear theory for the description of charged particle scattering by high-amplitude waves is due to the intrinsically nonlinear nature of such an interaction in the case of high enough amplitudes. ${ }^{39}$ In particular, the quasi-linear theory assumes that the time $T_{r}$ of the wave-particle resonant interaction does not depend on wave amplitudes and that it is determined by wave dispersion and the inhomogeneity of the background magnetic field. ${ }^{22,49,54}$ In this case, the small change of particle pitchangle $\Delta \alpha_{0}$ (or energy) due to a single act of scattering is proportional to the amplitude $B_{w}$ of the wave interacting with the particle (as the amplitude of the Lorentz force acting on that particle) multiplied by the time of interaction $T_{r}$. Due to the non-coherence of these many small changes $\Delta \alpha_{0}$, the average change is equal to zero, while the variance $\left\langle\left(\Delta \alpha_{0}\right)^{2}\right\rangle$ is about $\left(B_{w 0} T_{r}\right)^{2} \sim B_{w 0}^{2}$. This latter dependence corresponds to the quasi-linear approximation. However, for highamplitude waves the timescale of the wave-particle resonant interaction is controlled by the wave amplitude $T_{r} \sim B_{w 0}^{-1 / 2}$ (this is the so-called nonlinear regime of wave-particle interaction, see Refs. 20, 33, 36, and 41). As a result, we obtain a variance $\left\langle\left(\Delta \alpha_{0}\right)^{2}\right\rangle \sim B_{w 0}$ instead of $\sim B_{w 0}^{2}$ as predicted by the quasi-linear theory. Moreover, for high amplitude waves, there is a finite average change $\left\langle\Delta \alpha_{0}\right\rangle \neq 0$ providing particle non-diffusive drift in the pitch-angle (and energy) space. ${ }^{3,6}$ In such a case, the variation of particle pitch-angle and energy cannot be described as a diffusive process.

\section{DISCUSSION AND CONCLUSIONS}

In this paper, we have considered the scattering of relativistic electrons by fast magnetosonic waves. We have used a magnetic field configuration and system parameters typical for the Earth radiation belts. However, the obtained results can be generalized for applications in the magnetotail region, 
where strong magnetosonic emissions were recently observed. ${ }^{8,56}$ Magnetosonic waves represent a very convenient wave emission for testing the applicability of the quasi-linear approximation: being trapped within a narrow near-equatorial region, these waves interact with electrons locally, and thus, one does not need to take into account peculiarities of wave intensity and normal-angle distributions along magnetic field lines (such peculiarities are not well defined for whistler waves and represent a real challenge for modeling electron scattering in the radiation belts, see Refs. 1, 9, 25, 32, and 42). Using this natural advantage of the magnetosonic wave emission, we have been able to check the applicability of the quasi-linear approximation for describing electron scattering in the case of a discrete wave spectrum as well as for large wave amplitudes. Our study can be considered as an analog to the investigation presented in Ref. 47 for parallel whistler waves.

The data shown in Fig. 2 confirm the results obtained in Ref. 24: the quasi-linear approximation does not work properly for $\omega_{p e}<5$. In the inner magnetosphere, the range $\omega_{p e}<5$ is very important, since such values of $\omega_{p e}$ correspond to a large slice of the outer radiation belt at $L$-shells $\sim 3-5$, as well as to a significant part of the plasmasphere (see the empiric model of plasma density in Ref. 40). For $\omega_{p e}=3$ and $0.1-2 \mathrm{MeV}$ electrons, the magnitude of diffusion rates obtained from test particle modeling is $3-10$ times smaller than the predictions of the quasi-linear theory. This is an important result, because the contribution of magnetosonic waves is considered to be critical in the outer plasmasphere, where these waves may essentially control electron scattering in the absence of strong emissions of highfrequency lightning-generated or VLF whistler waves. ${ }^{5,30}$ Thus, a higher wave intensity is necessary to get an important contribution of magnetosonic waves. However, as Fig. 9 shows, the increase of diffusion rates with wave intensity is not linear. Thus, estimating the wave intensity sufficient to impact lifetimes of $\sim \mathrm{MeV}$ electrons inside the plasmasphere requires a separate investigation. It should also be mentioned that, in contrast to the radiation belts, the parameter $\omega_{p e}$ is usually larger than 5 in the Earth magnetotail (typical values are about $\omega_{p e} \approx 10$, see magnetotail parameters in Ref. 37). Thus, the quasi-linear approximation seems to be reliable enough for calculations of electron scattering by magnetosonic waves in the magnetotail.

The results obtained for large amplitude waves (see Fig. 9) demonstrate the natural problems of the quasi-linear approximation in the case of very intense wave emissions. Measured average amplitudes of fast magnetosonic waves in the radiation belts are about $10-50$ pT (see Refs. 28, 29, 31 , and 43) and thus, such values guarantee the applicability of the quasi-linear approximation. However, recent spacecraft measurements demonstrate the presence of magnetosonic waves with amplitudes about few hundred pTs. ${ }^{50,56}$ For these waves, our modeling predicts a significant overestimation of diffusion rates when using the quasi-linear approximation. In such a case, test particle simulations should better be used for accurately investigating electron scattering by intense waves.
The relationship between quasi-linear and nonlinear wave-particle interactions is determined by the wave amplitudes $^{39}$ and has not been studied yet extensively enough, because significant efforts are necessary to reproduce the transition between the regimes of purely nonlinear interaction (including phase bunching and particle trapping) and the regime of quasi-linear scattering. For most investigated chorus types of whistler-mode waves, the wave intensity and effective inhomogeneity of the system parameters vary significantly along magnetic field lines. ${ }^{2,36,41}$ Thus, nonlinear and quasi-linear regimes of wave-particle interaction can be encountered by the same particle at different locations along the field line. As a result, the effects of inhomogeneity seriously complicate the description of particle nonlinear/ quasi-linear scattering. ${ }^{6}$ Moreover, the additional effects of a wave emission localized within wave-packets ${ }^{4,48}$ and of the variation of wave frequency within such packets ${ }^{11,12,47}$ also make the analytical description of nonlinear wave-particle interaction rather non straightforward (recent spacecraft observations found similar frequency variation for magnetosonic waves as well ${ }^{13}$ ). Most of these problems can be easily overcome for magnetosonic waves interacting with particles locally within the equatorial region. In this case, the comparison between nonlinear and quasi-linear wave-particle interactions can be carried out almost analytically. We leave this study for future publications.

To conclude, in this paper, we have considered the scattering of relativistic electrons by magnetosonic waves in conditions typical for the radiation belts. Our results show that

- The discreteness of the magnetosonic wave emissions can influence significantly the wave-particle interaction only for extremely narrow wave normal angle distributions, while for realistic values of $\delta \theta$, the quasi-linear approximation still describes electron scattering rather well.

- For wave amplitudes larger than 300 pT, the nonlinear effects of wave-particle interaction start playing an important role. As a result, for such intense wave emissions, the quasi-linear approximation cannot describe wave-particle resonant interaction properly.

- In agreement with previous investigations, ${ }^{7,24}$ the quasilinear approximation can describe electron scattering by magnetosonic emissions localized within a $|\lambda|<3^{\circ}$ region only for a large enough plasma frequency $\left(\Omega_{p e} / \Omega_{c e} \sim 10\right)$.

The results obtained for high-amplitude waves open the door for further investigations of nonlinear electron scattering by magnetosonic waves.

\section{ACKNOWLEDGMENTS}

Authors are thankful to Professor M. Balikhin for pointing out the problem posed by discrete magnetosonic wave emissions observed in earth radiation belts. Work of A.A.V. was supported by Grant MK-1781.2014.2. Work by O.A. was performed under JHU/APL Contract No. 922613 (RBSP-EFW).

${ }^{1}$ O. Agapitov, A. Artemyev, V. Krasnoselskikh, Y. V. Khotyaintsev, D. Mourenas, H. Breuillard, M. Balikhin, and G. Rolland, J. Geophys. Res. 118, 3407-3420, doi:10.1002/jgra.50312 (2013). 
${ }^{2}$ O. Agapitov, A. Artemyev, D. Mourenas, V. Krasnoselskikh, J. Bonnell, O. Le Contel, C. M. Cully, and V. Angelopoulos, J. Geophys. Res. 119, 1606-1626, doi:10.1002/2013JA019223 (2014).

${ }^{3}$ J. M. Albert, Phys. Fluids B 5, 2744-2750 (1993).

${ }^{4}$ A. Artemyev, V. Krasnoselskikh, O. Agapitov, D. Mourenas, and G. Rolland, Phys. Plasmas 19, 122901 (2012).

${ }^{5}$ A. V. Artemyev, K. G. Orlova, D. Mourenas, O. V. Agapitov, and V. V. Krasnoselskikh, Ann. Geophys. 31, 1485-1490 (2013).

${ }^{6}$ A. V. Artemyev, A. A. Vasiliev, D. Mourenas, O. V. Agapitov, and V. V. Krasnoselskikh, Phys. Plasmas 21(10), 102903 (2014).

${ }^{7}$ J. Bortnik and R. M. Thorne, J. Geophys. Res. 115, 7213, doi:10.1029/ 2010JA015283 (2010).

${ }^{8}$ L. Chen, R. M. Thorne, V. K. Jordanova, and R. B. Horne, J. Geophys. Res. 115, 11222, doi:10.1029/2010JA015707 (2010).

${ }^{9}$ L. Chen, R. M. Thorne, W. Li, and J. Bortnik, J. Geophys. Res. 118, 1074-1088, doi:10.1029/2012JA018343 (2013).

${ }^{10}$ B. V. Chirikov, Particle Dynamics in Magnetic Traps, 1 st ed. (Consultants Bureau, New York, 1987), Vol. 13.

${ }^{11}$ A. G. Demekhov, V. Y. Trakhtengerts, M. Rycroft, and D. Nunn, Geomagn. Aeron. 49, 24-29, doi:10.1134/S0016793209010034 (2009).

${ }^{12}$ A. G. Demekhov, V. Y. Trakhtengerts, M. J. Rycroft, and D. Nunn, Geomagn. Aeron. 46, 711-716, doi:10.1134/S0016793206060053 (2006).

${ }^{13}$ H. S. Fu, J. B. Cao, Z. Zhima, Y. V. Khotyaintsev, V. Angelopoulos, O. Santolík, Y. Omura, U. Taubenschuss, L. Chen, and S. Y. Huang, Geophys. Res. Lett. 41, 7419-7426, doi:10.1002/2014GL061867 (2014).

${ }^{14}$ X. Fu, M. M. Cowee, R. H. Friedel, H. O. Funsten, S. P. Gary, G. B. Hospodarsky, C. Kletzing, W. Kurth, B. A. Larsen, K. Liu, E. A. MacDonald, K. Min, G. D. Reeves, R. M. Skoug, and D. Winske, J. Geophys. Res. 119, 8288, doi:10.1002/2014JA020364 (2014).

${ }^{15}$ S. P. Gary, K. Liu, D. Winske, and R. E. Denton, J. Geophys. Res. 115, 12209, doi:10.1029/2010JA015965 (2010).

${ }^{16}$ V. L. Ginzburg and A. A. Rukhadze, Waves in Magnetoactive Plasma, 2nd ed. (Nauka, Moscow, 1975).

${ }^{17}$ D. A. Gurnett, J. Geophys. Res. 81, 2765-2770, doi:10.1029/JA081i016p02765 (1976).

${ }^{18}$ R. B. Horne, R. M. Thorne, S. A. Glauert, N. P. Meredith, D. Pokhotelov, and O. Santolík, Geophys. Res. Lett. 34, 17107, doi:10.1029/ 2007GL030267 (2007).

${ }^{19}$ S. Irie and Y. Ohsawa, Phys. Plasmas 10, 1253-1261 (2003).

${ }^{20}$ V. I. Karpman, J. N. Istomin, and D. R. Shklyar, Plasma Phys. 16, 685-703 (1974)

${ }^{21}$ Y. Kasahara, H. Kenmochi, and I. Kimura, Radio Sci. 29, 751-767, doi:10.1029/94RS00445 (1994).

${ }^{22}$ C. F. Kennel and F. Engelmann, Phys. Fluids 9, 2377-2388 (1966).

${ }^{23}$ D. Krauss-Varban and N. Omidi, J. Geophys. Res. 96, 17715, doi:10.1029/ 91JA01545 (1991).

${ }^{24}$ J. Li, B. Ni, L. Xie, Z. Pu, J. Bortnik, R. M. Thorne, L. Chen, Q. Ma, S. $\mathrm{Fu}, \mathrm{Q}$. Zong, X. Wang, C. Xiao, Z. Yao, and R. Guo, Geophys. Res. Lett. 41, 4828-4834, doi:10.1002/2014GL060461 (2014).

${ }^{25}$ W. Li, D. Mourenas, A. Artemyev, O. Agapitov, J. Bortnik, J. Albert, R. M. Thorne, B. Ni, C. A. Kletzing, W. S. Kurth, and G. B. Hospodarsky, Geophys. Res. Lett. 41, 6063-6070, doi:10.1002/2014GL061260 (2014).

${ }^{26}$ K. Liu, S. P. Gary, and D. Winske, J. Geophys. Res. 116, A07212, doi:10.1029/2010JA016372 (2011).

${ }^{27}$ L. R. Lyons and D. J. Williams, Quantitative Aspects of Magnetospheric Physics (Springer, 1984).

${ }^{28}$ Q. Ma, W. Li, R. M. Thorne, and V. Angelopoulos, Geophys. Res. Lett. 40, 1895-1901, doi:10.1002/grl.50434 (2013).

${ }^{29}$ N. P. Meredith, R. B. Horne, and R. R. Anderson, J. Geophys. Res. 113, 6213, doi:10.1029/2007JA012975 (2008).
${ }^{30}$ N. P. Meredith, R. B. Horne, S. A. Glauert, D. N. Baker, S. G. Kanekal, and J. M. Albert, J. Geophys. Res. 114, 3222, doi:10.1029/2008JA013889 (2009).

${ }^{31}$ D. Mourenas, A. V. Artemyev, O. V. Agapitov, and V. Krasnoselskikh, J. Geophys. Res. 118, 3096-3112, doi:10.1002/jgra.50349 (2013).

${ }^{32}$ D. Mourenas, A. V. Artemyev, O. V. Agapitov, and V. Krasnoselskikh, J. Geophys. Res. 119, 2775-2796, doi:10.1002/2013JA019674 (2014).

${ }^{33}$ D. Nunn, J. Plasma Phys. 6, 291 (1971).

${ }^{34}$ F. Němec, O. Santolík, K. Gereová, E. Macúšová, Y. de Conchy, and N. Cornilleau-Wehrlin, Planet. Space Sci. 53, 291-298 (2005).

${ }^{35}$ F. Němec, O. Santolík, Z. Hrbackova, and N. Cornilleau-Wehrlin, J. Geophys. Res. 120(3), 1620-1632, doi:10.1002/2014JA020814 (2015).

${ }^{36}$ Y. Omura, Y. Katoh, and D. Summers, J. Geophys. Res. 113, 4223, doi:10.1029/2007JA012622 (2008).

${ }^{37}$ A. A. Petrukovich, A. V. Artemyev, I. Y. Vasko, R. Nakamura, and L. M. Zelenyi, Space Sci. Rev. 188, 311-337 (2015).

${ }^{38}$ C. T. Russell, R. E. Holzer, and E. J. Smith, J. Geophys. Res. 75, 755, doi:10.1029/JA075i004p00755 (1970).

${ }^{39}$ V. D. Shapiro and R. Z. Sagdeev, Phys. Rep. 283, 49-71 (1997).

${ }^{40}$ B. W. Sheeley, M. B. Moldwin, H. K. Rassoul, and R. R. Anderson, J. Geophys. Res. 106, 25631-25642, doi:10.1029/2000JA000286 (2001).

${ }^{41}$ D. Shklyar and H. Matsumoto, Surv. Geophysics 30, 55-104 (2009).

${ }^{42}$ Y. Y. Shprits and B. Ni, J. Geophys. Res. 114, 11205, doi:10.1029/ 2009JA014223 (2009).

${ }^{43}$ Y. Y. Shprits, A. Runov, and B. Ni, J. Geophys. Res. 118, 648-652, doi:10.1002/jgra.50108 (2013).

${ }^{44}$ T. H. Stix, The Theory of Plasma Waves (McGraw-Hill, New York, 1962).

${ }^{45}$ X. Tao and J. Bortnik, Nonlinear Process. Geophys. 17, 599-604 (2010).

${ }^{46}$ X. Tao, J. Bortnik, J. M. Albert, K. Liu, and R. M. Thorne, Geophys. Res. Lett. 38, 6105, doi:10.1029/2011GL046787 (2011).

${ }^{47}$ X. Tao, J. Bortnik, J. M. Albert, and R. M. Thorne, J. Geophys. Res. 117, 10205, doi:10.1029/2012JA017931 (2012).

${ }^{48}$ X. Tao, J. Bortnik, J. M. Albert, R. M. Thorne, and W. Li, J. Geophys. Res. 119, 8848-8857, doi:10.1002/2014JA020022 (2014).

${ }^{49}$ V. Y. Trakhtengerts, Geomagn. Aeron. 6, 827-836 (1966).

${ }^{50}$ B. T. Tsurutani, B. J. Falkowski, J. S. Pickett, O. P. Verkhoglyadova, O. Santolik, and G. S. Lakhina, J. Geophys. Res. 119, 964-977, doi:10.1002/ 2013JA019284 (2014).

${ }^{51}$ D. L. Turner, S. G. Claudepierre, J. F. Fennell, T. P. O’Brien, J. B. Blake, C. Lemon, M. Gkioulidou, K. Takahashi, G. D. Reeves, S. Thaller, A. Breneman, J. R. Wygant, W. Li, A. Runov, and V. Angelopoulos, Geophys. Res. Lett. 42, 2079-2087, doi:10.1002/2015GL063225 (2015).

${ }^{52}$ C. Yang, Y. He, L. Zhang, Q. Zhou, and F. Xiao, Plasma Phys. Controlled Fusion 55(6), 062001 (2013).

${ }^{53}$ A. A. Vedenov and R. Z. Sagdeev, "Some properties of a plasma with an anisotropic ion velocity distribution in a magnetic field," in Plasma Physics and the Problem of Controlled Thermonuclear Reactions, edited by M. A. Leontovich (Pergamon Press, 1961), Vol. 3. p. 332.

${ }^{54}$ A. A. Vedenov, E. Velikhov, and R. Sagdeev, Nucl. Fusion 2, 465475 (1962).

${ }^{55}$ L. B. Wilson, D. G. Sibeck, A. W. Breneman, O. L. Contel, C. Cully, D. L. Turner, V. Angelopoulos, and D. M. Malaspina, J. Geophys. Res. 119, 6475-6495, doi:10.1002/2014JA019930 (2014).

${ }^{56}$ M. Zhou, B. Ni, S. Huang, X. Deng, M. Ashour-Abdalla, Y. Nishimura, Z. Yuan, Y. Pang, and H. Li, J. Geophys. Res. 119, 4335-4347, doi:10.1002/ 2014JA019796 (2014).

${ }^{57}$ Q. Zhou, F. Xiao, C. Yang, S. Liu, C. A. Kletzing, W. S. Kurth, G. B. Hospodarsky, H. E. Spence, G. D. Reeves, H. O. Funsten, J. B. Blake, D. N. Baker, and J. R. Wygant, J. Geophys. Res. 119, 9125-9133, doi:10.1002/2014JA020481 (2014). 